\title{
Inverted Electron-Hole Alignment in InAs-GaAs Self-Assembled Quantum Dots
}

\author{
P. W. Fry, ${ }^{1}$ I. E. Itskevich, ${ }^{2,1}$ D. J. Mowbray, ${ }^{1}$ M. S. Skolnick, ${ }^{1}$ J. J. Finley, ${ }^{1}$ J. A. Barker, ${ }^{3}$ E. P. O’Reilly, ${ }^{3}$ L. R. Wilson, ${ }^{1}$ \\ I. A. Larkin, ${ }^{1}$ P. A. Maksym, ${ }^{4}$ M. Hopkinson, ${ }^{5}$ M. Al-Khafaji, ${ }^{5}$ J. P. R. David, ${ }^{5}$ A. G. Cullis,${ }^{5}$ G. Hill, ${ }^{5}$ and J. C. Clark ${ }^{5}$ \\ ${ }^{1}$ Department of Physics and Astronomy, University of Sheffield, Sheffield S3 7RH, United Kingdom \\ ${ }^{2}$ Institute of Solid State Physics, Russian Academy of Sciences, Chernogolovka, Moscow district, 142432, Russia \\ ${ }^{3}$ Department of Physics, University of Surrey, Guildford GU2 5XH, United Kingdom \\ ${ }^{4}$ Department of Physics, University of Leicester, Leicester LE1 7RH, United Kingdom \\ ${ }^{5}$ Department of Electronic and Electrical Engineering, University of Sheffield, Sheffield S1 3JD, United Kingdom
}

(Received 5 April 1999)

\begin{abstract}
New information on the electron-hole wave functions in InAs-GaAs self-assembled quantum dots is deduced from Stark effect spectroscopy. Most unexpectedly it is shown that the hole is localized towards the top of the dot, above the electron, an alignment that is inverted relative to the predictions of all recent calculations. We are able to obtain new information on the structure and composition of buried quantum dots from modeling of the data. We also demonstrate that the excited state transitions arise from lateral quantization and that tuning through the inhomogeneous distribution of dot energies can be achieved by variation of electric field.
\end{abstract}

PACS numbers: 73.61. $-\mathrm{r}, 68.90 .+\mathrm{g}, 73.50 . P z, 78.66 .-\mathrm{w}$

Self-assembled InAs-GaAs quantum dots (QDs) provide nearly ideal examples of zero-dimensional semiconductor systems [1] and are hence of considerable contemporary interest for the study of new physics and potential device applications. However, very little is known experimentally about the nature of the QD carrier wave functions and their response to applied fields. Numerous calculations of the electronic structure of QDs have been performed [2-5], but in the absence of definitive structural information they assume idealized QD shapes, usually pyramidal [6]. However there is evidence that in many cases the dots more closely approximate to lens shaped [7], and may also contain significant concentrations of Ga [8], rather than being pure InAs. In view of the uncertainties in shape and composition, the applicability of the calculated electronic structure to real dots must at best be regarded as approximate at the present time.

Consequently, experimental information on the nature of the wave functions is urgently required, to provide a reliable guide to theory, and a firm basis for the interpretation of experiments. In this paper we demonstrate that photocurrent spectroscopy under electric field $F$ provides important, new information on the carrier wave functions, and by comparison with theory, on the composition, shape and effective height of the dots. We show that the QDs possess a permanent dipole moment, implying a finite spatial separation of the electron and hole for $F=0$. Contrary to the predictions of all recent calculations, we demonstrate that the holes are localized above the electrons in the QDs. This "inverted" alignment can only be explained by assuming nonuniform $\mathrm{Ga}$ incorporation in the nominally InAs QDs. As a result of our work the extensive previous theoretical modeling of the electronic structure of InAs QDs will need to be reexamined.

Two types of dots were studied, both grown by molecular-beam epitaxy on (001) GaAs substrates at
$500{ }^{\circ} \mathrm{C}$. The first type (samples $A-C$ ) was deposited at 0.01 monolayers per second $(\mathrm{ML} / \mathrm{s})$ to give a density $\approx 1.5 \times 10^{9} \mathrm{~cm}^{-2}$, base size $18 \mathrm{~nm}$, and height $8.5 \mathrm{~nm}$ [Fig. 1(a)], as determined from transmission electron microscopy (TEM). The second type (sample $D$ ) had a higher deposition rate of $0.4 \mathrm{ML} / \mathrm{s}$, resulting in a density $\approx 5 \times 10^{10} \mathrm{~cm}^{-2}$ and size $15 \times 3.5 \mathrm{~nm}$. The asymmetric shaped QDs, sitting on an $\approx 1 \mathrm{ML}$ thick wetting layer, have their apex oriented along the growth direction. Buried single dot layers were grown within the

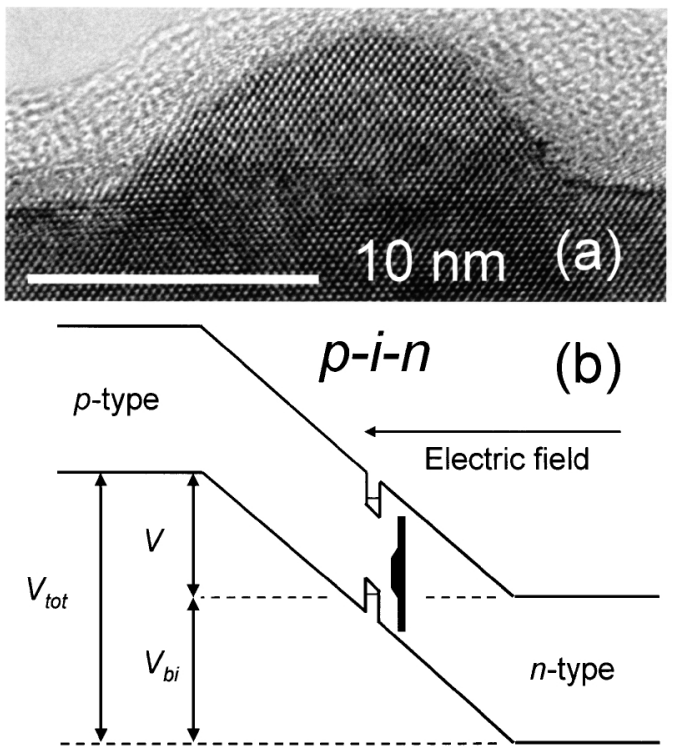

FIG. 1. (a) Cross-sectional TEM micrograph of an uncapped slow growth rate quantum dot. The buried dots studied in the photocurrent experiments showed similar size and shape in TEM but gave less clear images due to the presence of the GaAs matrix. (b) Schematic band diagram of the $p-i-n$ device under reverse bias $V$. 
intrinsic region (width $d$ ) of either $p-i-n$ or $n-i-p$ structures, allowing fields $F$ up to $300 \mathrm{kV} / \mathrm{cm}$ to be applied either parallel or antiparallel to the growth direction. Applying a reverse bias to a $p-i-n$ structure ( $p$ region at surface) results in $F$ pointing from substrate to surface [Fig. 1(b)]. For $n-i-p$ the field direction is reversed. The total field is given by $F=\left(V+V_{\mathrm{bi}}\right) / d$, where $V$ is the applied voltage and $V_{\mathrm{bi}} \approx 1.5 \mathrm{~V}$ is the built-in voltage $(d=0.3 \mu \mathrm{m}$ for samples $A-C$ and $0.5 \mu \mathrm{m}$ for sample $D$ ). Monochromated white light $\left(\approx 3 \mathrm{~mW} / \mathrm{cm}^{2}\right.$, corresponding to extremely low dot carrier occupancies) was used for excitation. The photocurrent was measured with lock-in techniques, using $400 \mu \mathrm{m}$ diameter, annular contact mesas.

Photocurrent spectra, for unpolarized light incident on the surface of the sample, as a function of reverse bias from 0 to $8 \mathrm{~V}$ ( $F$ from 50 to $320 \mathrm{kV} / \mathrm{cm}$ ), are shown in Figs. 2(a) and 2(b) for sample $A(p-i-n)$ at temperatures $T$ of 5 and $200 \mathrm{~K}$, respectively. At $200 \mathrm{~K}$, four well defined features are observed from 1.1 to $1.3 \mathrm{eV}$, arising from interband transitions between the QD confined electron and hole levels. Similar spectra are seen at $5 \mathrm{~K}$, though at low fields the interband transitions are quenched, and a signal is observed only from the InAs wetting layer $(1.41 \mathrm{eV})$ and the GaAs barriers (1.5 eV) [Fig. 2(a), inset]. The temperature dependence arises from the change in the dominant carrier escape mechanism (carrier escape enables the photoexcited carriers to give rise to a photocurrent) from tunneling at low $T$ to thermal excitation at high $T$. With increasing field, at all $T$, all of the QD transitions shift strongly to lower energy [by $30 \mathrm{meV}$ at $8 \mathrm{~V}$ $(\approx 300 \mathrm{kV} / \mathrm{cm})]$, the signature of the quantum confined Stark effect [9], but without any qualitative change in the form of the spectra.

Photocurrent spectra for light incident on a cleaved edge of a sample containing QDs grown under the same conditions as samples $A, B$, and $C$, but with the $i$ region surrounded by $\mathrm{AlGaAs}$ cladding layers to provide in-plane light guiding are presented in the inset to Fig. 2(b) [10]. Spectra are shown for light polarized either in the growth plane $\left(E_{\|}\right)$or perpendicular to it $\left(E_{\perp}\right)$ [10]. The QD transitions are seen to be strongly $E_{\|}$polarized, demonstrating that they have predominantly heavy-hole character [11].

The ground state transition energies at $200 \mathrm{~K}$ for samples $A-D$ are plotted in Fig. 3(a) as a function of $F$, calculated using $V_{\mathrm{bi}}=1.5 \mathrm{~V}$. Positive field corresponds to reverse bias for $p$ - $i-n$ structures. We focus attention first on samples $B(p-i-n)$ and $C(n-i-p)$ [12]. Their transition energies exhibit a marked asymmetry about $F=0$, with the maximum obtained for a nonzero field of $-90 \mathrm{kV} / \mathrm{cm}$. This asymmetry implies that the QDs have a permanent dipole moment $p$. A nonzero Stark shift at $F=0$ of the ground state transition has also been observed in the photoluminescence (PL) of InAlAs-AlGaAs QDs [13] over the limited field range of $\pm 60 \mathrm{kV} / \mathrm{cm}$ (PL is unavoidably quenched at higher fields).

The field dependence of the transition energies $E$ in Fig. 3(a) can be described to a very reasonable approxima-

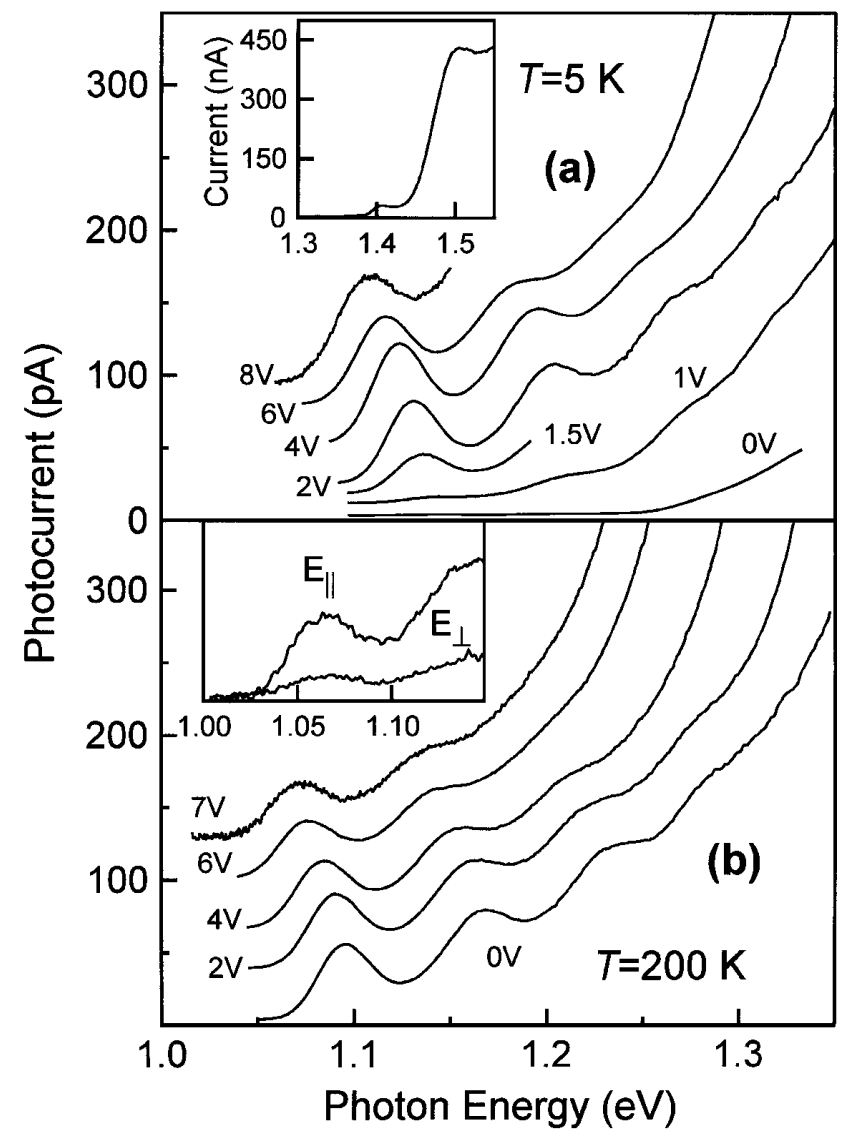

FIG. 2. Photocurrent spectra of sample $A$, as a function of applied reverse bias, for temperatures of $5 \mathrm{~K}$ (a) and $200 \mathrm{~K}$ (b). The inset to (a) contains a photocurrent spectrum $(V=0$, $T=5 \mathrm{~K}$ ) showing the wetting layer and GaAs absorption. The inset to (b) shows QD spectra at $0 \mathrm{~V}$ (field $50 \mathrm{kV} / \mathrm{cm}$ ) for light incident on the edge of low growth rate sample at $300 \mathrm{~K}$, polarized either within $\left(E_{\|}\right)$or perpendicular to $\left(E_{\perp}\right)$ the growth plane. At high energy the spectra are unpolarized showing the absence of any instrumental effects.

tion by the expression $E=E_{0}+p F+\beta F^{2}$ [the solid lines in Fig. 3(a)], where $E_{0}$ is the energy at $F=0$, the second term arises from the nonzero dipole moment $p$, and the third term arises from polarization of the dots in the applied field (the quantum confined Stark effect). By fitting to the data for samples $B$ and $C$, a value of $p=$ $(7 \pm 2) \times 10^{-29} \mathrm{Cm}$ is determined, corresponding to an electron-hole separation of $r=4 \pm 1 \AA$ (from $p=e r$ ).

Theoretical modeling of self-assembled QDs predicts a permanent dipole moment due to their asymmetric shape [2]. However, the experimentally determined direction of the dipole is very surprising. The maximum transition energy occurs for $F$ in the direction from apex to base, corresponding to electron (hole) attraction to the apex (base) of the dots. This implies that the electron charge density distribution lies below that of the hole at $F=0$, with the dipole pointing from base to apex. This result is in contrast to that predicted by all accurate modeling [2-5] of InAs QDs which predicts hole localization towards the base of the dots, below the electrons. This calculated alignment occurs for pure InAs dots of any shape for which the 


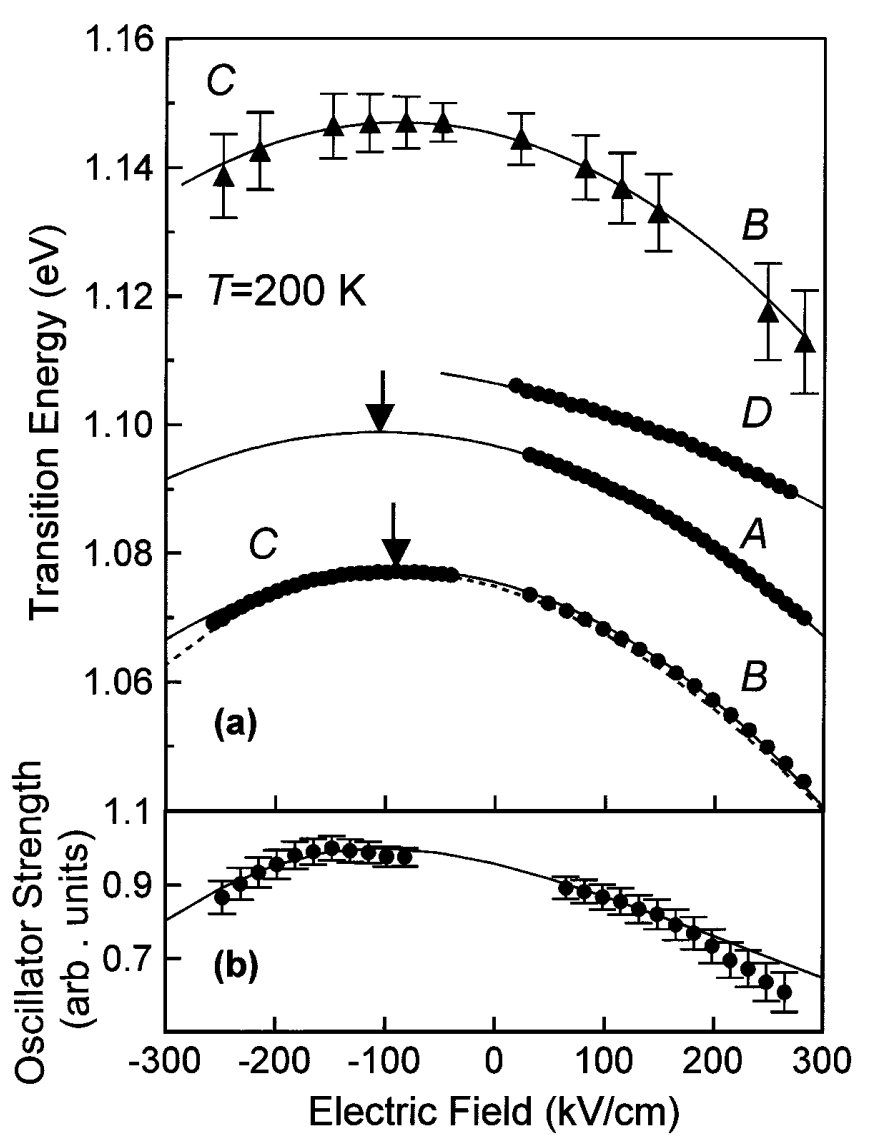

FIG. 3. (a) Ground state transition energies (solid circles) as a function of electric field for samples $A-D$ at a temperature of $200 \mathrm{~K}$. The solid curves are a fit to $E=E_{0}+p F+\beta F^{2}$. The arrows indicate the maxima of the transition energies. The data for sample $C$ have been rigidly shifted by $7 \mathrm{meV}$ [12]. The dashed curve is a theoretical fit to the data for samples $B$ and $C$ using the model described in the text. The triangles to higher energy show the variation with field of the first excited state transition energy for samples $B$ and $C$. The line through these points is the same parabola drawn through the $B, C$ ground state results. (b) Ground state photocurrent intensities with field (solid circles) for samples $B$ and $C$ and comparison with theoretical variation of electron-hole overlap squared (solid line).

lateral size decreases from base to apex (e.g., the pyramidal shape in Refs. [2-5]). It results from the strain-induced form of the valence band edge profile [3] (the strain acts to localize the holes in the widest region of the dots) and the difference in the effective mass ratios along the growth direction $\left(m_{h h}^{*} \gg m_{e}^{*}\right)$.

To determine dot structural parameters to reverse the electron and hole alignment, and to fit the data of Fig. 3(a), envelope function calculations, treating the electrons and holes with separate one-band Hamiltonians, have been performed. The strain distribution for a given dot shape was calculated using a Green's function technique which provides an analytical expression in the form of a Fourier series for the strain tensor [14]. Band gaps and offsets were calculated using model solid theory [15], including hydrostatic strain effects; the heavy-hole Hamiltonian included the spatial variation of the biaxial strain deformation potential and the directional dependence of the heavy-hole mass [as discussed earlier, the edge photocurrent measurements in the Fig. 2(b) inset demonstrate that the lowest transitions involve heavy-hole levels, justifying an assumption of the theory]. Inverse carrier masses, determined using three-band $\mathbf{k} \cdot \mathbf{p}$ theory, and band offsets were assumed to vary linearly with composition.

For pure InAs pyramidal dots our calculations give good agreement with previous theories [2-5], with the hole wave function below that of the electron. We find reversal of this alignment is only possible if two conditions are fulfilled. Firstly, the dots must have a graded $\operatorname{In}_{1-x} \mathrm{Ga}_{x}$ As composition, with $x$ decreasing from base to apex (the holes tend to be localized in the region with the largest In composition). Secondly, it is also necessary to severely truncate the pyramid to achieve a dipole of the correct sign, since strain localizes the hole strongly below the electron until the truncation factor is greater than $\approx 0.6$ [16].

The best fit to the experimental data for samples $B$ and $C$ is shown as the dashed line in Fig. 3(a). This was obtained using a pyramid of base length $w=15.5 \mathrm{~nm}$, height $22 \mathrm{~nm}$, of which the top $75 \%$ is truncated [see Fig. 1(b)] to give an actual height $b$ of $5.5 \mathrm{~nm}$, and $x$ varying linearly from 0.5 at the base to zero at the top surface. The quadratic component $\beta$ of the energy shift is determined principally by $b$, the dipole $p$ by the grading and the degree of truncation, and the absolute energy $E_{0}$ mainly by $b$ and $w$. In addition to fitting the data very well, we emphasize that the shape and size ( $b$ and $w$ ) employed represent a good approximation to those obtained from structural measurements [Fig. 1(a)]. Furthermore, any shape wider at the base than the apex (including full pyramids) of constant composition, or full pyramids with linear grading, never has a dipole of the correct sign. We thus obtain new information by optical methods on the structure and composition of buried QDs which is very difficult to obtain from structural studies. Our conclusions regarding the composition of buried InAs dots are consistent with results of STM investigations on uncapped dots, which found evidence for $\mathrm{Ga}$ in-diffusion at the present growth temperature $\left(500^{\circ} \mathrm{C}\right)$ [8], and for In segregation [17].

Data for devices $A$ and $D$ (slow and fast growth rates respectively) in Fig. 3(a) also exhibit a maximum transition energy for a negative field, showing that a permanent dipole with hole above electron is a general property of our QDs. Furthermore, the same sign of asymmetry of the Stark effect has also been reported in AlInAs/AlGaAs self-assembled QDs [13] supporting our findings. In addition, we also note that the size of the quadratic component of the Stark shift for the slow growth rate samples $(A-C)$ is larger than that of the faster growth rate sample $D$, consistent with the smaller height of the dots in these samples.

The variation of the ground state photocurrent intensity with field at $200 \mathrm{~K}$ is shown in Fig. 3(b) for samples $B$ and $C$. It shows a qualitatively very similar variation to that of the transition energies in Fig. 3(a). The marked decrease with increasing positive field arises from the 


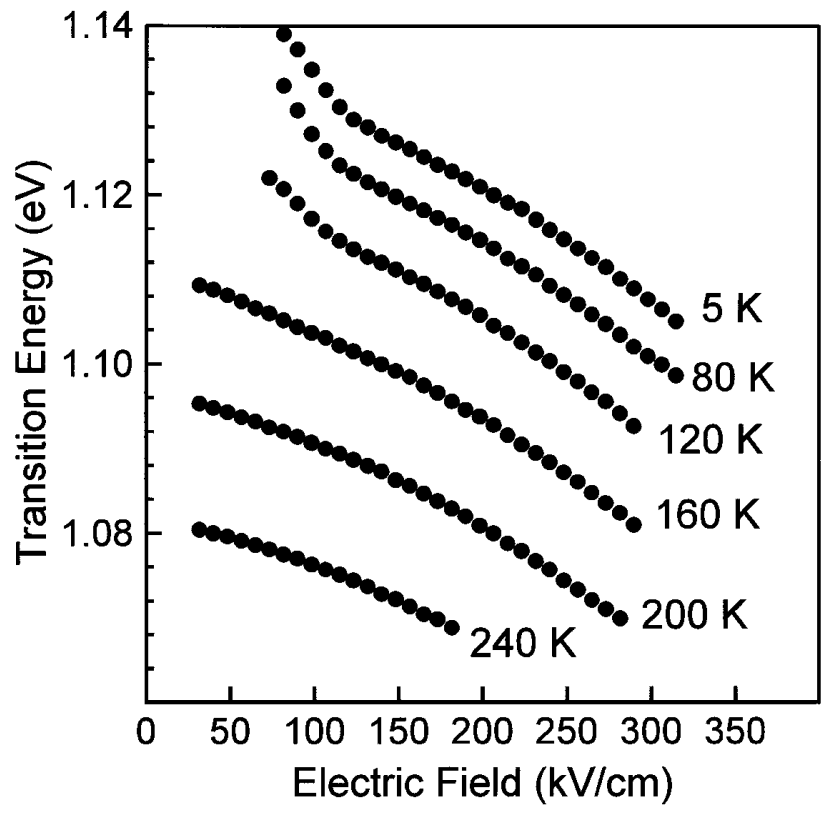

FIG. 4. Transition energies as a function of field for sample $A$ at temperatures from 5 to $240 \mathrm{~K}$.

decrease in electron-hole overlap with $F$. It is notable that the maximum photocurrent intensity for the $n-i-p$ sample occurs at $-140 \mathrm{kV} / \mathrm{cm}$, in good agreement with the field of maximum transition energy in Fig. 3(a) where the electron-hole overlap is expected to be greatest. The results are also reproduced very well by our calculations of the $e-h$ overlap squared with field [the solid line in Fig. 3(b)] using the same parameters as for the fits of Fig. 3(a), providing further clear support for the overall model.

In Fig. 2(b), the energy separations and relative intensities of the transitions can be seen to be independent of field [18], in strong contrast to the behavior for quantum wells [9]. This behavior is consistent with the excited state splittings arising from lateral quantization since, to first order, the response to $F$ along the growth direction $z$ is determined by quantization along $z$.

The data in Fig. 3(a) were obtained at $200 \mathrm{~K}$. The variation of the ground state peak position with $F$ from 240 to $5 \mathrm{~K}$ is plotted in Fig. 4. At low $T$, and $F$ less than $\approx 120 \mathrm{kV} / \mathrm{cm}$, there is increasing departure from the parabolic behavior of Fig. 3(a). This deviation arises from selective tunneling from the inhomogeneous distribution of dot energies (at low $T$ tunneling is the dominant carrier escape mechanism). At low $F$, only carriers excited in the highest energy part of the distribution escape, and the transition energy occurs above that expected by extrapolation from higher field. As $F$ is increased, tunneling also becomes possible from the lower energy parts of the distribution, until at $120 \mathrm{kV} / \mathrm{cm}$ all carriers escape and the observed energy corresponds to the mean of the distribution. We are thus able to tune through the inhomogeneously broadened dot distribution by varying applied bias.

In summary, we have demonstrated that InAs-GaAs self-assembled quantum dots possess a permanent dipole moment. The dipole moment is expected from the asymmetric shape of the dots but its sign opposite to that predicted by all recent calculations. In combination with theoretical modeling we have deduced the effective height of the dots and the electron-hole vertical separation, and have demonstrated that the nominally InAs dots contain significant concentrations of Ga. In light of these results, much previous theoretical modeling will need to be reexamined. We have also demonstrated that selective carrier escape from within the inhomogeneous distribution of dot energies can be achieved.

We thank A. D. Andreev for useful discussions and acknowledge EPSRC and the Russian Foundation for Fundamental Research.

[1] D. Bimberg, M. Grundmann, and N. N. Ledentsov, Quantum Dot Heterostructures (Wiley, New York, 1998).

[2] M. Grundmann, O. Stier, and D. Bimberg, Phys. Rev. B 52, 11969 (1995).

[3] M. Cusack, P. R. Briddon, and M. Jaros, Phys. Rev. B 54, R2300 (1996).

[4] C. Pryor, Phys. Rev. B 57, 7190 (1998). This reference (8-band $\mathbf{k} \cdot \mathbf{p}$ theory) provides a summary of most earlier theoretical work.

[5] J. Kim, L.-W. Wang, and A. Zunger, Phys. Rev. B 57, R9408 (1998).

[6] S. Ruvimov et al., Phys. Rev. B 51, 14766 (1995).

[7] D. S. L. Mui, D. Leonard, L. A. Coldren, and P. M. Petroff, Appl. Phys. Lett. 66, 1620 (1995).

[8] P. B. Joyce et al., Phys. Rev. B 58, R15 981 (1998).

[9] D. A. B. Miller et al., Phys. Rev. B 32, 1043 (1985).

[10] This sample showed extremely similar surface photocurrent spectra to those of samples $A, B$, and $C$.

[11] See, e.g., G. Bastard, Wave Mechanics Applied to Semiconductor Heterostructures (Wiley, New York, 1988), p. 248.

[12] A small shift of $7 \mathrm{meV}$ to higher energy has been applied to the results of sample $C$, in order to obtain a continuous variation of the peak positions between positive and negative electric fields. Samples $B$ and $C$ were grown consecutively to obtain the minimum run-to-run variation in dot parameters between samples.

[13] S. Raymond et al., Phys. Rev. B 58, R13 415 (1998).

[14] A. D. Andreev, J.R. Downes, D. A. Faux, and E. P. O’Reilly, J. Appl. Phys. 86, 297 (1999).

[15] M. P. M. C. Krijn, Semicond. Sci. Technol. 6, 27 (1991).

[16] Piezoelectric fields have a negligible effect on the dipole moment and the ground state energies $(<0.3 \mathrm{meV}$ shift). This arises since there is no piezoelectric effect along the $\langle 100\rangle$ growth direction and, due to symmetry about planes containing $\langle 100\rangle$, fields from the sides of the dots cancel to small values $(<10 \mathrm{kV} / \mathrm{cm})$ in the central $50 \AA$, where the wave functions are concentrated [see J. H. Davies, J. Appl. Phys. 84, 1358 (1998)].

[17] N. Grandjean, J. Massies, and O. Tottereau, Phys. Rev. B 55, R10 189 (1997).

[18] The field independence of the energy separation of the ground and first excited state transitions can also be seen by inspection of Fig. 3(a) for samples $B$ and $C$. 